\title{
Corn stover pretreatment by ionic liquid and glycerol mixtures with their density, viscosity, and thermogravimetric properties
}

Joan G. Lynam*, Genica I. Chow, Phillip L. Hyland, and Charles J. Coronella

Department of Chemical \& Materials Engineering, University of Nevada, Reno, 1664 N. Virginia St., MS0170,

Reno, NV 89557, USA

"Corresponding author. E-mail address: lynam.joan@gmail.com (J. Lynam).

Supporting Information, 13 pages

Table S1. Corn stover (CS) composition by fiber analysis

Figure S1. Yields of glucose as \% of original cellulose and xylose as \% of original xylan versus enzymatic hydrolysis time

Table S2 Vibration assignment for cellulose, lignin, and hemicellulose and references

Figure S2 Sample spectra of raw and pretreated corn stover

Table S3 Peak heights for vibrations $896 \mathrm{~cm}^{-1}, 1162 \mathrm{~cm}^{-1}, 1425 \mathrm{~cm}^{-1}, 1515 \mathrm{~cm}^{-1}, 1600 \mathrm{~cm}^{-1}$, and $1730 \mathrm{~cm}^{-1}$

Figure $\mathbf{S 3}$ Viscosity of binary mixtures

Table S4 Viscosities ( П / Pa s) of (a) ([C2mim][OAc] (IL) + Glycerol binary mixtures) and (b) $([\mathrm{C} 2 \mathrm{mim}][\mathrm{O} 2 \mathrm{CH}](\mathrm{IL})+$ Glycerol binary mixtures). Mass \% used.

Table S5 Vogel-Fulcher-Tammann (VFT) equation parameters

Table S6 Peak differential scanning calorimetry temperatures for glycerol vaporization and decomposition of ILs and IL-glycerol mixtures

\section{References}


Table S1 Corn stover (CS) composition by fiber analysis

\begin{tabular}{lllll}
\hline Hemicellulose (\%) & Cellulose (\%) & Lignin (\%) & Extractives (\%) & Ash (\%) \\
\hline $\mathbf{2 7 . 2}$ & 30.7 & 9.8 & 27.2 & 5.1 \\
\hline
\end{tabular}

Fiber analysis was performed using the NDF-ADF-ADL Van Soest method ${ }^{1}$ prior to rinsing. Ash content was determined by heating to $575{ }^{\circ} \mathrm{C}$ for $12 \mathrm{~h}$ in a muffle furnace before and after rinsing. Rinsing reduced ash from $8.2 \%$ to $5.1 \%$, indicating that some loose dirt had been removed. Other components values are increased to reflect the mass of ash removal. Xylan constituted approximately $80 \%$ of the hemicellulosic structural sugar of the raw CS, with galactan and arabinan comprising the balance. 


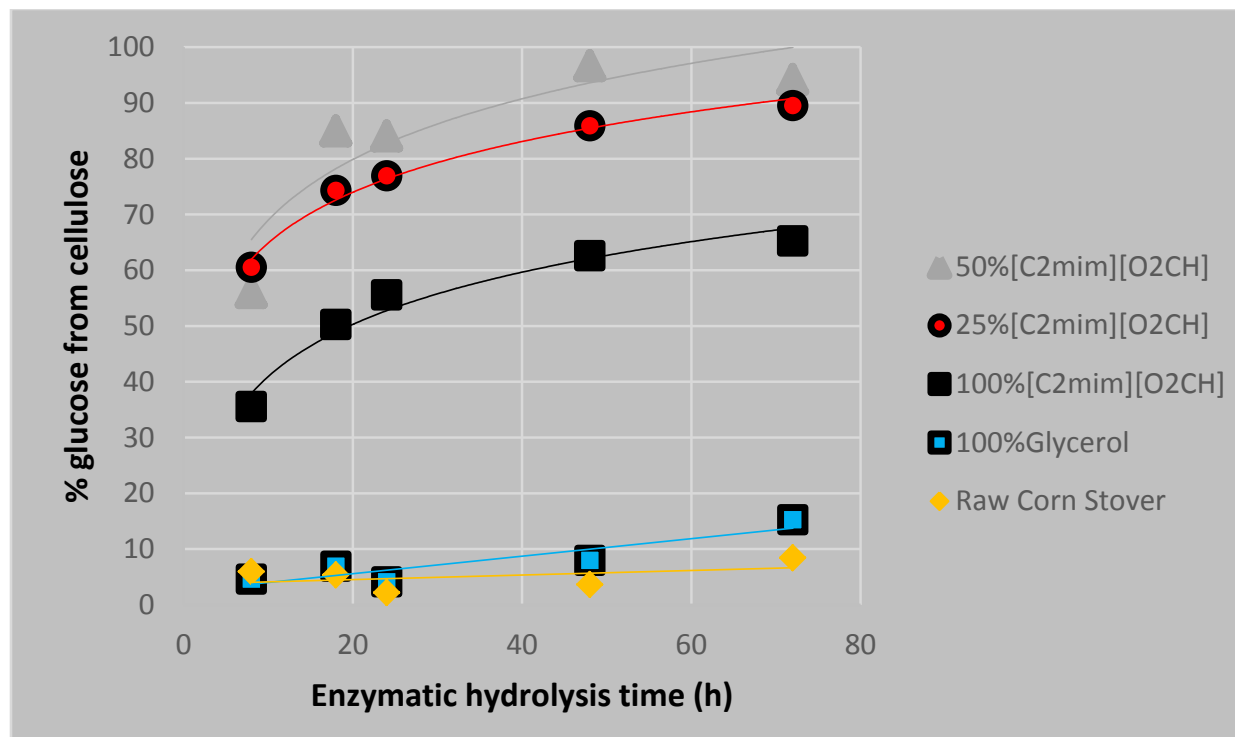

a)

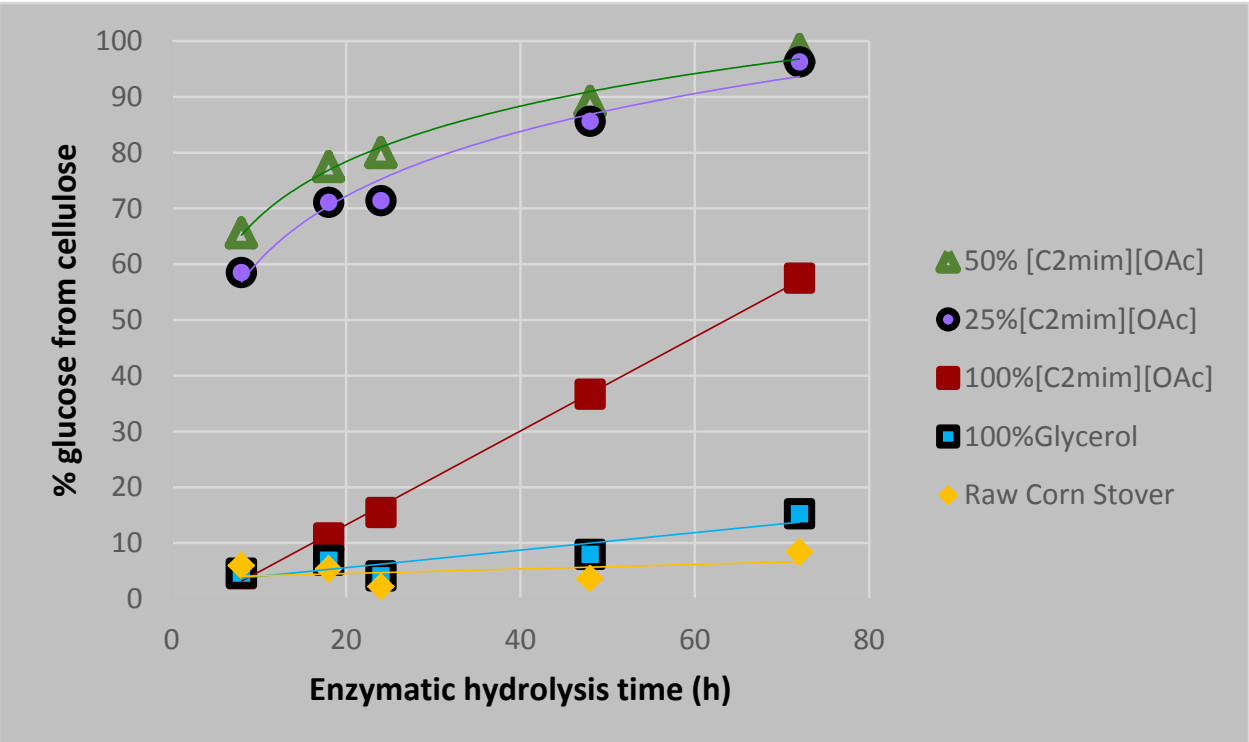

b) 


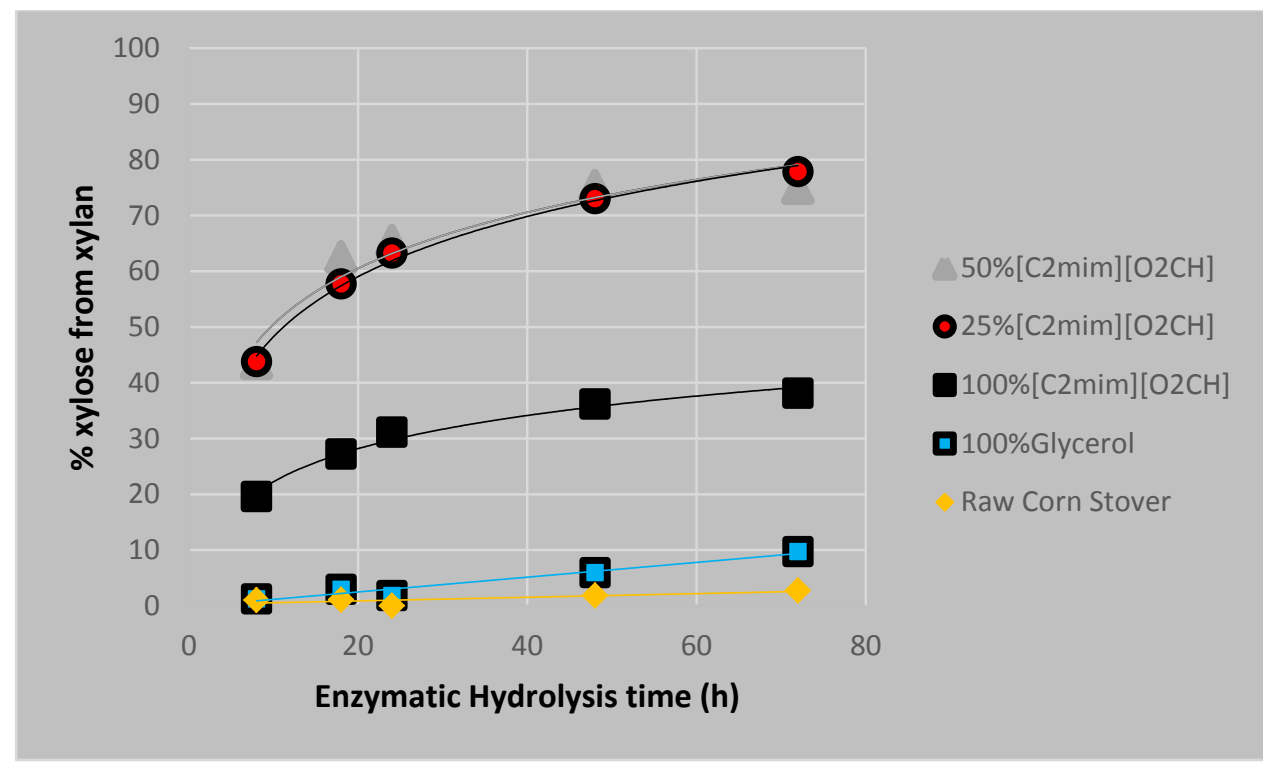

c)

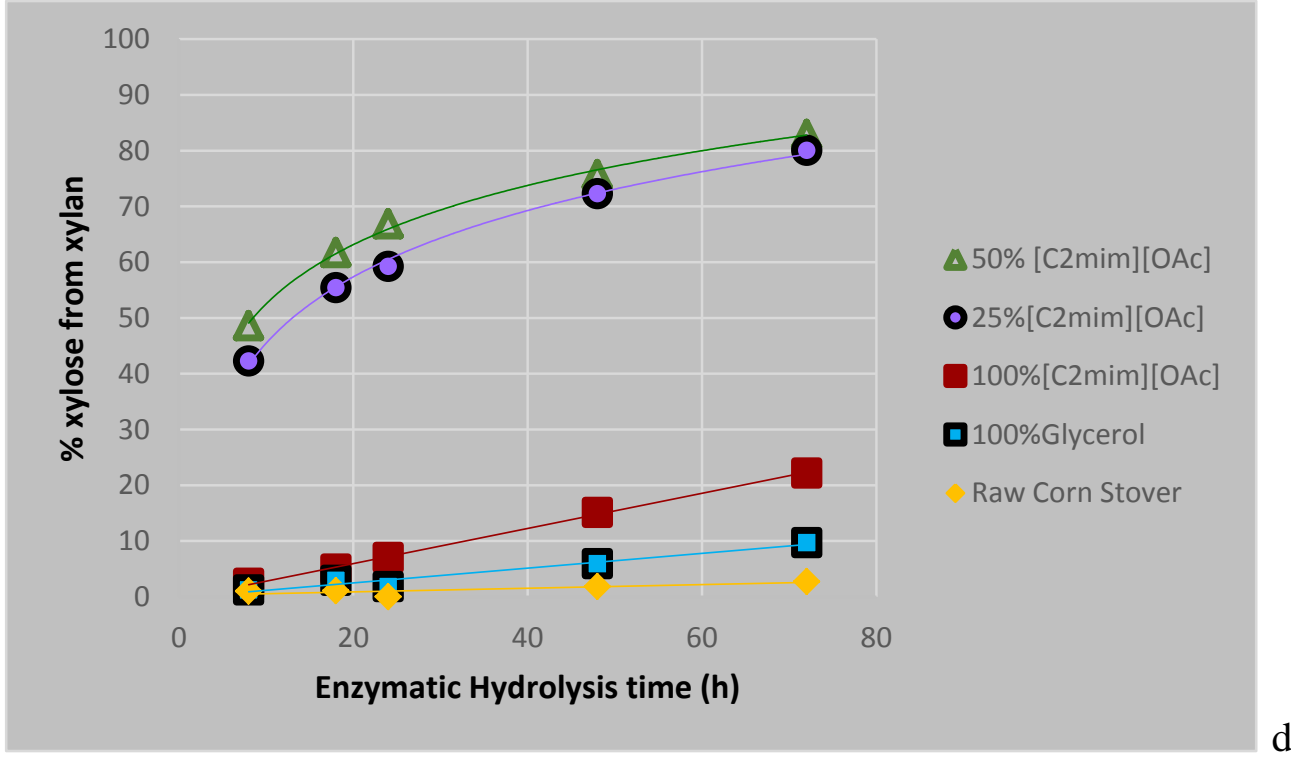

d)

Figure S1. Yields of glucose as \% of original cellulose for glycerol, raw CS and a) $[\mathrm{C} 2 \mathrm{mim}][\mathrm{O} 2 \mathrm{CH}]$ pretreatments, b) $[\mathrm{C} 2 \mathrm{mim}][\mathrm{OAc}]$ pretreatments vs enzymatic hydrolysis time; and yields of xylose as \% of original xylan for glycerol, raw $\mathrm{CS}$ and c) $[\mathrm{C} 2 \mathrm{mim}][\mathrm{O} 2 \mathrm{CH}]$ pretreatments, d) [C2mim][OAc] pretreatments vs enzymatic hydrolysis time 
Table S2 Vibration assignment for cellulose, lignin, and hemicellulose and references

\begin{tabular}{|c|c|c|}
\hline $\begin{array}{l}\text { Vibration } \\
\left(\mathbf{c m}^{-1}\right)\end{array}$ & Component & Reference \\
\hline 896 & $\begin{array}{l}\mathrm{C}_{1}-\mathrm{H} \text { deformation with a ring vibration contribution for } \\
\text { amorphous cellulose }\end{array}$ & $\left(\right.$ Raj et al., 2015) ${ }^{2}$ \\
\hline 1162 & $\mathrm{C}-\mathrm{O}-\mathrm{C}$ vibration in cellulose & $(\text { Wang et al., 2015) })^{3}$ \\
\hline 1425 & Crystalline cellulose & (Raj et al., 2015) ${ }^{2}$ \\
\hline 1515 & Lignin aromatic ring skeletal stretch & $\begin{array}{l}\text { (Raj et al., 2015,Wang et al., } \\
2015 ;)^{2-3}\end{array}$ \\
\hline 1600 & $\begin{array}{l}\text { Lignin } \mathrm{C}=\mathrm{O} \text { stretching conjugated to the aromatic ring, } \\
\text { aromatic ring vibration }\end{array}$ & $\begin{array}{l}\text { (Li et al., 2015; Lin et al., } \\
2015)^{4-5}\end{array}$ \\
\hline 1730 & $\begin{array}{l}\text { Ester acetyl, uronic acid groups in hemicelluloses; } \\
\text { ester group of ferulic and p-coumaric acids in lignin; } \\
\text { hemicelluose-lignin linkages }\end{array}$ & $\begin{array}{l}\text { (Costa et al., 2015; Lopes et } \\
\text { al., 2015) }\end{array}$ \\
\hline
\end{tabular}




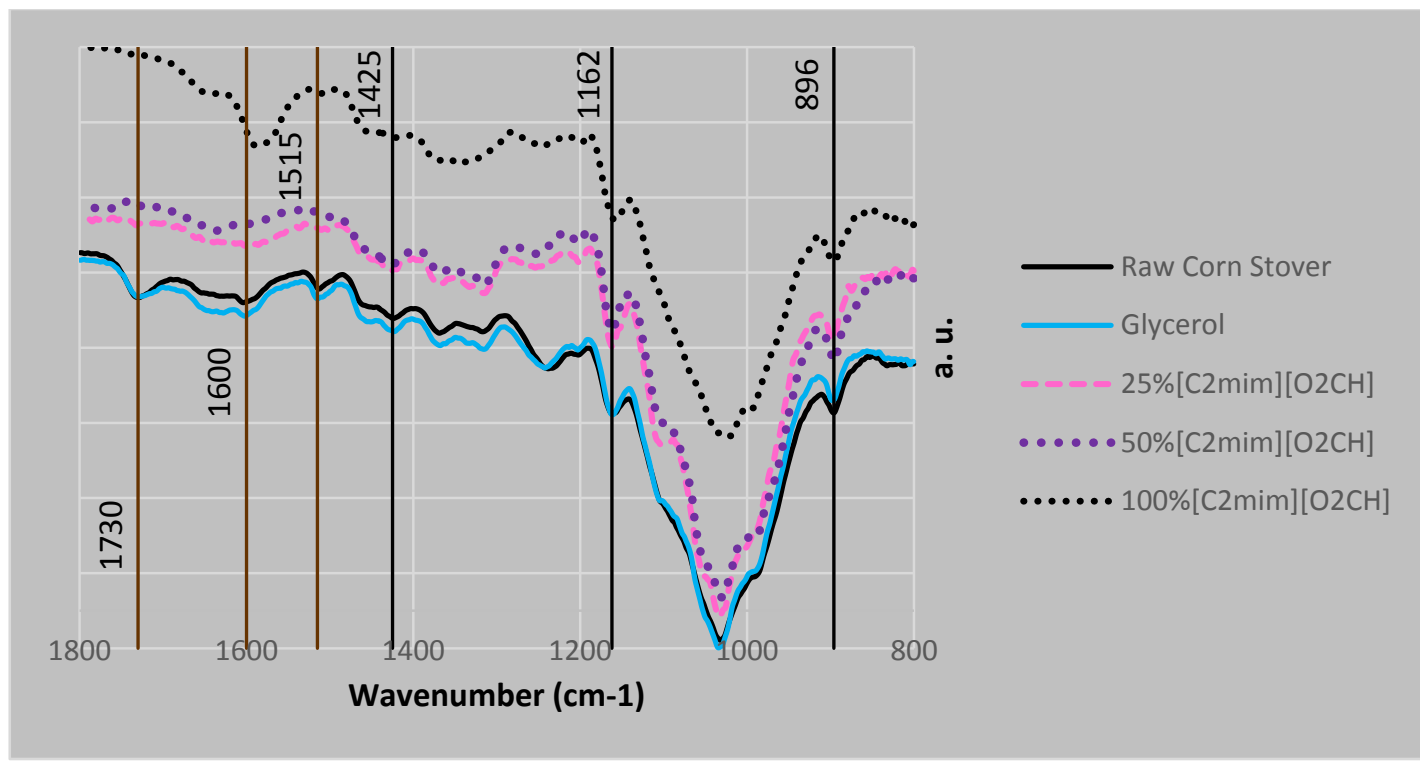

a)

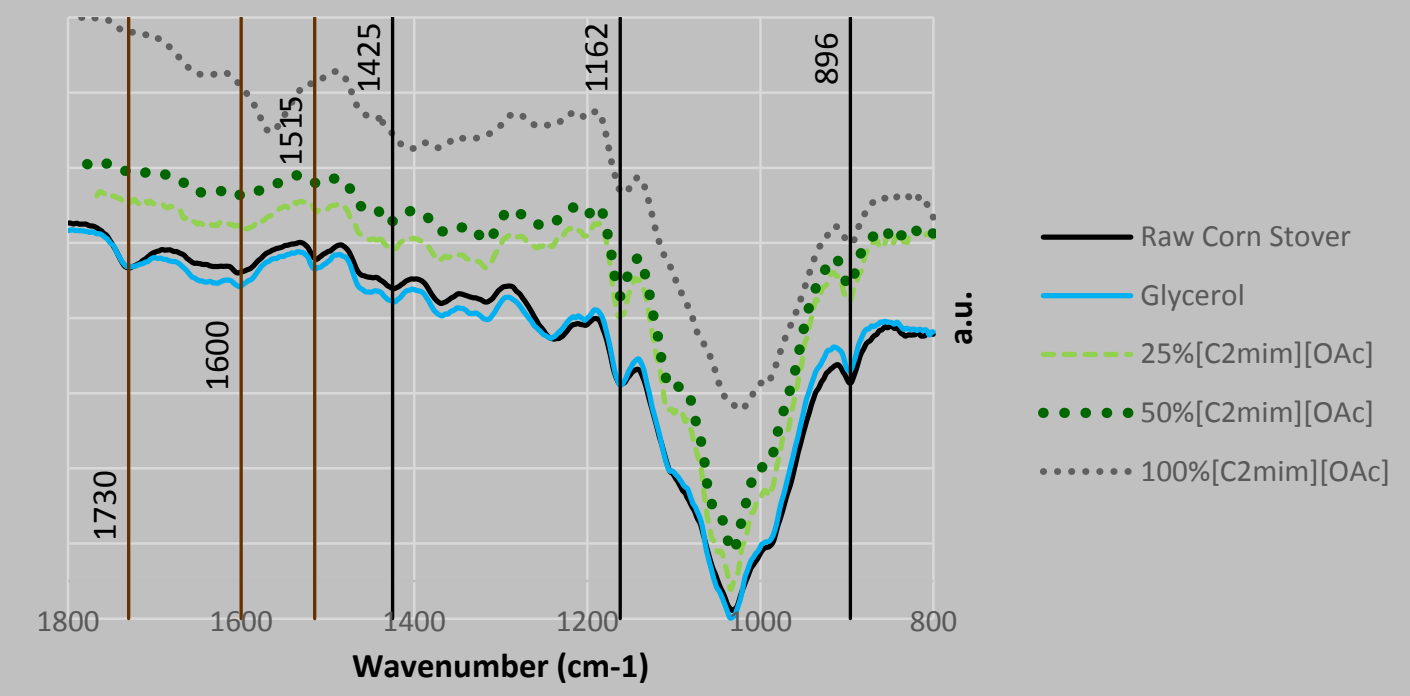

b)

Figure S2 Sample spectra of raw and pretreated corn stover a) [C2mim][O2CH], b) $[\mathrm{C} 2 \mathrm{mim}][\mathrm{OAc}]$

Three spectra of a given condition were averaged for each line, and spectra were normalized using the $2300 \mathrm{~cm}-1$ plateau baseline and the $1030 \mathrm{~cm}-1$ peak, since it has lignin, cellulose, and hemicellulose components. ${ }^{9}$ All peak heights were measured from the lower wavelength plateau. 
Table S3 Peak heights for vibrations $896 \mathrm{~cm}^{-1}, 1162 \mathrm{~cm}^{-1}, 1425 \mathrm{~cm}^{-1}, 1515 \mathrm{~cm}^{-1}, 1600 \mathrm{~cm}^{-1}$, and $1730 \mathrm{~cm}^{-1}$

\begin{tabular}{ccccccccc}
\hline $\begin{array}{l}\text { Vibration } \\
\text { cm-1 }\end{array}$ & Raw CS & Glycerol & $\begin{array}{l}25 \% \\
{[\mathrm{C} 2 \mathrm{mim}]} \\
{[\mathrm{OAc}]}\end{array}$ & $\begin{array}{l}50 \% \\
{[\mathrm{C} 2 \mathrm{mim}]} \\
{[\mathrm{OAc}]}\end{array}$ & $\begin{array}{l}100 \% \\
{[\mathrm{C} 2 \mathrm{mim}]} \\
{[\mathrm{OAc}]}\end{array}$ & $\begin{array}{l}25 \% \\
{[\mathrm{C} 2 \mathrm{mim}]} \\
{[\mathrm{O} 2 \mathrm{CH}]}\end{array}$ & $\begin{array}{l}50 \% \\
{[\mathrm{C} 2 \mathrm{mim}]} \\
{[\mathrm{O} 2 \mathrm{CH}]}\end{array}$ & $\begin{array}{l}100 \% \\
{[\mathrm{C} 2 \mathrm{mim}]} \\
{[\mathrm{O} 2 \mathrm{CH}]}\end{array}$ \\
\hline $\mathbf{8 9 6}$ & 15.2 & 12.4 & 16.3 & 14.9 & 9.0 & 15.6 & 19.9 & 9.0 \\
$\mathbf{1 1 6 2}$ & 3.9 & 6.4 & 9.7 & 10.5 & 13.6 & 10.4 & 10.3 & 15.2 \\
$\mathbf{1 4 2 5}$ & 2.6 & 3.5 & 2.8 & 2.3 & & 3.2 & 3.5 & \\
$\mathbf{1 5 1 5}$ & 3.8 & 4.2 & 1.9 & 1.6 & & 1.2 & 0.2 & \\
$\mathbf{1 6 0 0}$ & 8.1 & 10.0 & 4.2 & 5.4 & & 2.1 & 0.4 & \\
$\mathbf{1 7 3 0}$ & 4.9 & 1.5 & 0.5 & 0.1 & & 0.9 & 0.3 & \\
\hline
\end{tabular}




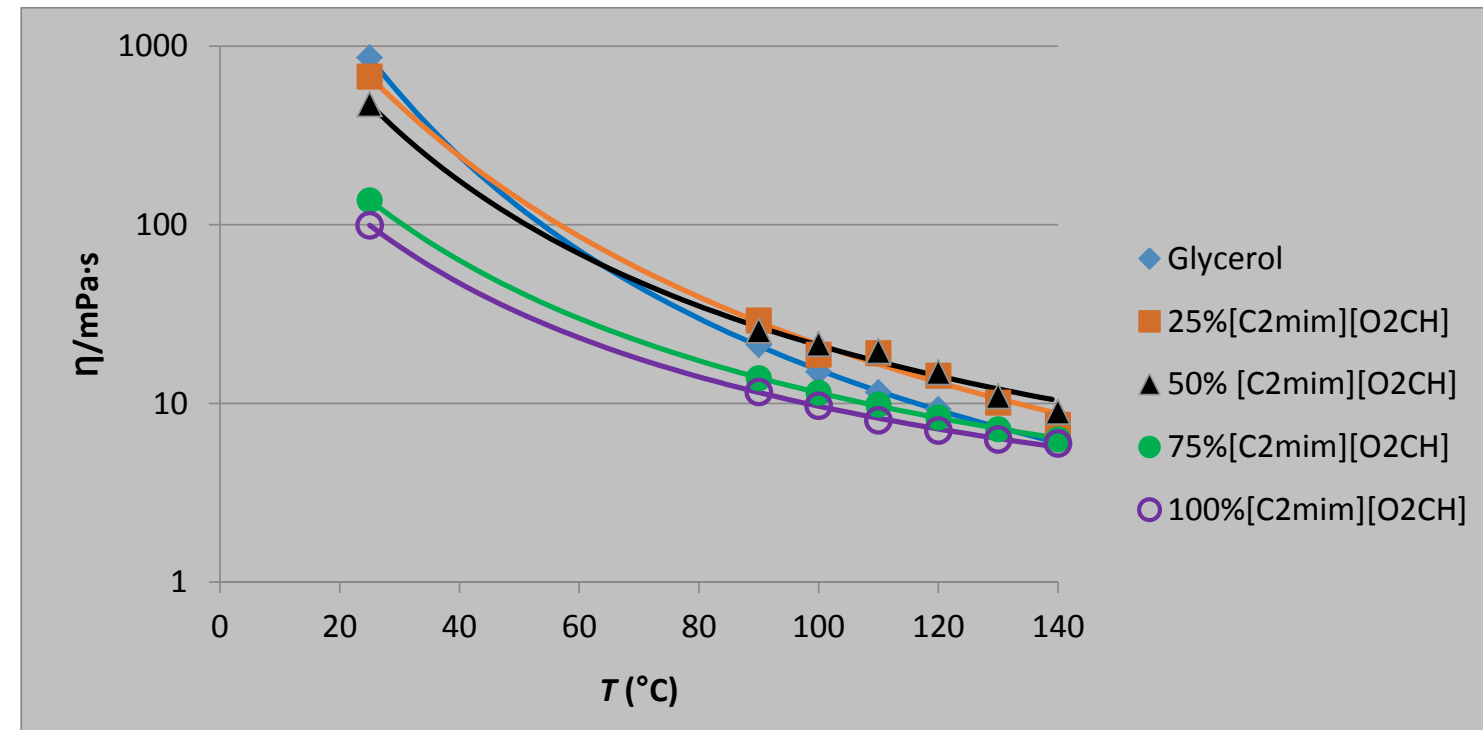

a)

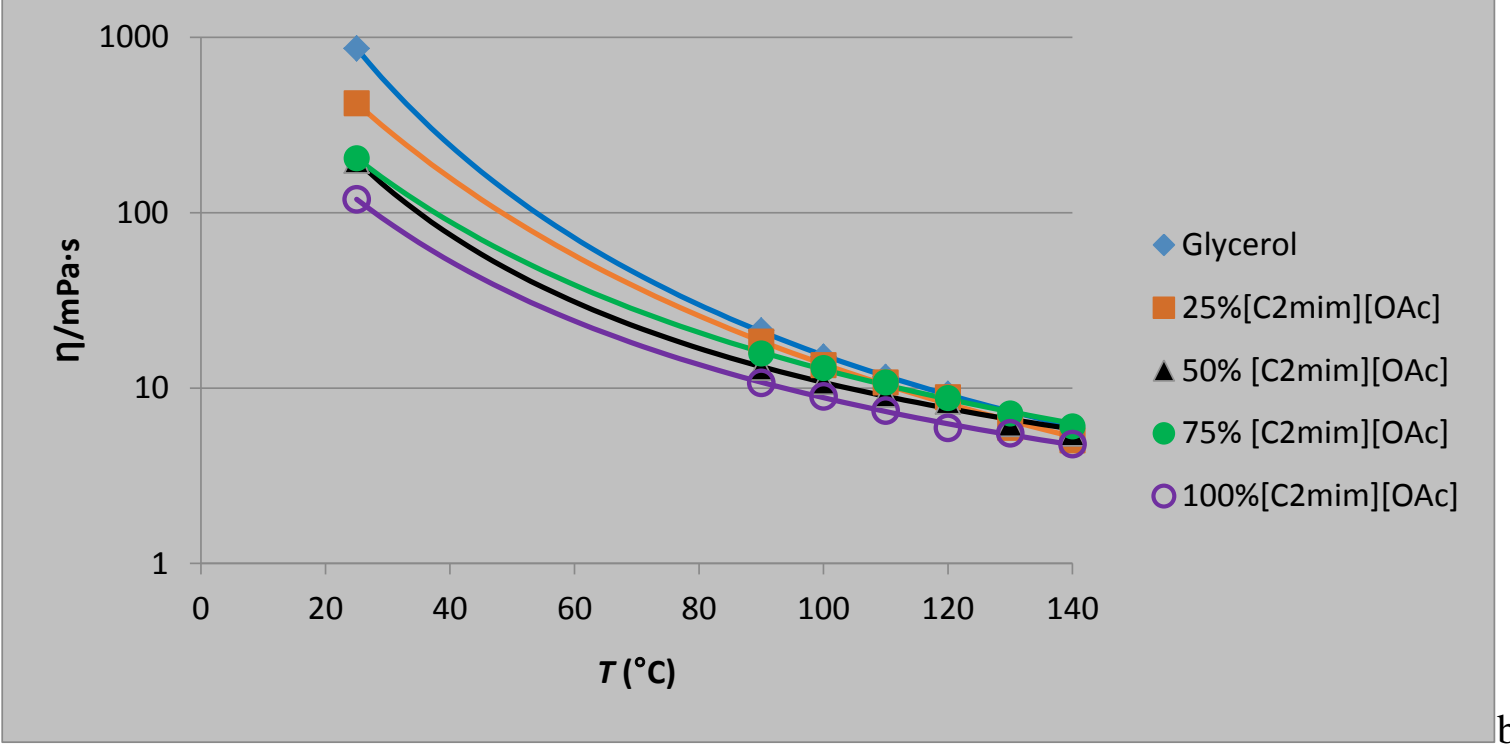

Figure S3 Viscosity of the a) ([C2mim] [O2CH] (1) + Glycerol (2)) and b) ([C2mim][OAc] (1) + Glycerol (2)) binary mixtures in the temperature range from $25{ }^{\circ} \mathrm{C}$ to $140{ }^{\circ} \mathrm{C}$ for different IL

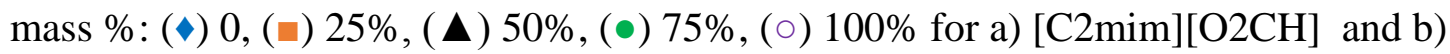
[C2mim][OAc]. The lines represent the VFT type fitting of the experimental data with parameters reported in Table S4 in the supplementary data. 
The IL $[\mathrm{C} 2 \mathrm{mim}][\mathrm{O} 2 \mathrm{CH}]$ apparently has a viscosity similar to that of glycerol at biomass processing temperatures, so there is little increase in viscosity with glycerol addition.

The mole fractions of $0.164,0.371$, and 0.638 of [C2mim][O2CH], correspond to 25 mass $\%, 50$ mass $\%$, and $75 \mathrm{mass} \%$ of $[\mathrm{C} 2 \mathrm{mim}][\mathrm{O} 2 \mathrm{CH}]$, as shown in the legend. Viscosities are slightly higher for $[\mathrm{C} 2 \mathrm{mim}][\mathrm{O} 2 \mathrm{CH}]$ than for $[\mathrm{C} 2 \mathrm{mim}][\mathrm{OAc}]$ (Figure $\mathrm{S} 3$ ), possibly due to the fact that the $[\mathrm{O} 2 \mathrm{CH}]$ anion is more compact than $[\mathrm{OAc}]$ and so the $[\mathrm{C} 2 \mathrm{mim}][\mathrm{O} 2 \mathrm{CH}]$ salt is more tightly coordinated. For this reason $[\mathrm{C} 2 \mathrm{mim}][\mathrm{O} 2 \mathrm{CH}]$ has a melting point near $56{ }^{\circ} \mathrm{C},{ }^{10}$ which is above ambient conditions, although it exhibits supercooling behavior. ${ }^{11}$ In contrast, [C2mim][OAc] has a melting point below room temperature, since its larger anion cannot be as tightly coordinated as $[\mathrm{O} 2 \mathrm{CH}]$.

$[\mathrm{C} 2 \mathrm{mim}][\mathrm{OAc}]$ and glycerol have same viscosity trends as $[\mathrm{C} 2 \mathrm{mim}][\mathrm{O} 2 \mathrm{CH}]$ and glycerol (Figure S3). The mole fractions shown, $0.153,0.351$, and 0.619 of [C2mim][OAc], correspond to 25 mass $\%, 50$ mass $\%$, and 75 mass $\%$ of [C2mim][OAc], as shown in the caption and legend. Viscosities are highly divergent under ambient conditions, but relatively similar at typical biomass processing temperatures. 
Table S4 Viscosities ( $\rceil$ / Pa $\cdot s)$ of (a) ([C2mim][OAc] (IL) + Glycerol binary mixtures) and (b) ([C2mim] $[\mathrm{O} 2 \mathrm{CH}](\mathrm{IL})+$ Glycerol binary mixtures). Mass \% used.

(a) ([C2mim] $[\mathrm{OAc}]$ (IL) + Glycerol binary mixtures)

\begin{tabular}{rrrrrr}
\hline $\mathbf{T} /{ }^{\circ} \mathbf{C}$ & $\mathbf{0 \%} \mathbf{~ I L}$ & $\mathbf{2 5 \%} \mathbf{~ I L}$ & $\mathbf{5 0 \%} \mathbf{~ I L}$ & $\mathbf{7 5 \%} \mathbf{~ I L}$ & $\mathbf{1 0 0 \%} \mathbf{~ I L}$ \\
\hline $\mathbf{2 5}$ & 0.8731 & 0.4264 & 0.2000 & 0.2067 & 0.1239 \\
$\mathbf{2 5}$ & 0.8554 & 0.4149 & 0.1957 & 0.2016 & 0.1149 \\
$\mathbf{9 0}$ & 0.02122 & 0.01848 & 0.01284 & 0.01549 & 0.01023 \\
$\mathbf{9 0}$ & 0.02132 & 0.01830 & 0.01323 & 0.01600 & 0.01122 \\
$\mathbf{1 0 0}$ & 0.01499 & 0.01347 & 0.01074 & 0.01297 & 0.008954 \\
$\mathbf{1 0 0}$ & 0.01515 & 0.01356 & 0.01094 & 0.01304 & 0.008987 \\
$\mathbf{1 1 0}$ & 0.01153 & 0.01071 & 0.009036 & 0.01072 & 0.007573 \\
$\mathbf{1 1 0}$ & 0.01157 & 0.01084 & 0.009286 & 0.01081 & 0.007328 \\
$\mathbf{1 2 0}$ & 0.009506 & 0.008847 & 0.008427 & 0.008681 & 0.005935 \\
$\mathbf{1 2 0}$ & 0.009039 & 0.008888 & 0.008327 & 0.008853 & 0.005934 \\
$\mathbf{1 3 0}$ & 0.006564 & 0.005869 & 0.006148 & 0.007202 & 0.005569 \\
$\mathbf{1 3 0}$ & 0.007164 & 0.005982 & 0.006332 & 0.007138 & 0.005479 \\
$\mathbf{1 4 0}$ & 0.006442 & 0.005041 & 0.005476 & 0.005930 & 0.004809 \\
$\mathbf{1 4 0}$ & 0.007032 & 0.005025 & 0.005512 & 0.006170 & 0.004779 \\
\hline
\end{tabular}

(b) $([\mathrm{C} 2 \mathrm{mim}][\mathrm{O} 2 \mathrm{CH}](\mathrm{IL})+$ Glycerol binary mixtures

\begin{tabular}{|c|c|c|c|c|c|}
\hline $\mathbf{T} /{ }^{\circ} \mathbf{C}$ & $0 \%$ IL & $25 \%$ IL & $50 \%$ IL & $75 \% \mathrm{IL}$ & $100 \%$ IL \\
\hline 25 & 0.8731 & 0.6601 & 0.4648 & 0.1373 & 0.1013 \\
\hline 25 & 0.8554 & 0.6942 & 0.4818 & 0.1370 & 0.09759 \\
\hline 90 & 0.02122 & 0.03200 & 0.02549 & 0.01362 & 0.01167 \\
\hline 90 & 0.02132 & 0.02622 & 0.02555 & 0.01409 & 0.01154 \\
\hline 100 & 0.01499 & 0.01904 & 0.02244 & 0.01139 & 0.009661 \\
\hline 100 & 0.01515 & 0.01844 & 0.02064 & 0.0 & 0.009775 \\
\hline 110 & 0.01153 & 0.02123 & 0.02020 & 0.009753 & 0.007974 \\
\hline 110 & 0.01157 & 0.01702 & 0.01898 & 0.009920 & 0.008129 \\
\hline 120 & 0.009506 & 0.01337 & 0.01516 & 0.008333 & 0.007025 \\
\hline 120 & 0.009039 & 0.01527 & 0.01472 & 0.008375 & 0.007086 \\
\hline 130 & 0.006564 & 0.01082 & 0.01123 & 0.007147 & 0.006247 \\
\hline 130 & 0.007164 & 0.009419 & 0.01072 & 0.007181 & 0.006319 \\
\hline 140 & 0.006442 & 0.008403 & 0.009208 & 0.006264 & 0.005905 \\
\hline 140 & 0.007032 & 0.006756 & 0.008743 & 0.006321 & 0.006037 \\
\hline
\end{tabular}




\section{Table S5}

Vogel-Fulcher-Tammann (VFT) equation parameters (a) ([C2mim][OAc] (IL) + Glycerol binary mixtures) and (b) ([C2mim] [O2CH] (IL) + Glycerol binary mixtures). Mass $\%$ used. $^{12}$

$\eta=\eta_{0} \exp \left(D T_{0} /\left(T-T_{0}\right)\right)$

\begin{tabular}{|c|c|c|c|c|c|}
\hline VFT parameters & \multicolumn{5}{|c|}{ [C2mim][OAc] } \\
\hline (a) & $0 \% \mathrm{IL}$ & $25 \% \mathrm{IL}$ & $50 \% \mathrm{IL}$ & $75 \% \mathrm{IL}$ & $100 \% \mathrm{IL}$ \\
\hline$\eta_{0}(\mathrm{~Pa}-\mathrm{sec})$ & 0.0001312 & 0.0000455 & 0.0005899 & 0.0002627 & 0.0003598 \\
\hline$D$ (unitless) & 3.73 & 6.59 & 1.95 & 3.62 & 2.59 \\
\hline T0 /K & 209.40 & 173.19 & 223.27 & 193.05 & 206.09 \\
\hline \multirow[t]{2}{*}{ (b) } & \multicolumn{5}{|c|}{$[\mathrm{C} 2 \mathrm{mim}][\mathrm{O} 2 \mathrm{CH}]$} \\
\hline & $0 \% \mathrm{IL}$ & $25 \% \mathrm{IL}$ & $50 \% \mathrm{IL}$ & $75 \%$ IL & $100 \% \mathrm{IL}$ \\
\hline$\eta_{0}(\mathrm{~Pa}-\mathrm{sec})$ & 0.0001312 & 0.0001477 & 0.0005832 & 0.0005543 & 0.0006876 \\
\hline D (unitless) & 3.73 & 4.80 & 2.74 & 2.44 & 1.98 \\
\hline T0 /K & 209.40 & 190.03 & 211.53 & 206.54 & 213.21 \\
\hline
\end{tabular}

The Angell strength parameter $D$ is large $(D \approx 20-100)$ for "strong" liquids where the viscosity approaches Arrhenius temperature dependence. The parameter $D$ is smaller $(D \approx 3-5)$ for "fragile" liquids. ${ }^{13}$ As can be seen from table S2, the Angell parameter for both the pure ionic liquids is close to 2 , while $D$ is 4 for glycerol, so all three are in the "fragile" range. ${ }^{14}$ 
Table S6 Peak differential scanning calorimetry temperatures for glycerol vaporization and decomposition of ILs and IL-glycerol mixtures and temperature at which $10 \%$ of the mass remaining after a $105^{\circ} \mathrm{C}$ hold is lost.

\begin{tabular}{|c|c|c|}
\hline Composition & $\begin{array}{l}\text { T }\left({ }^{\circ} \mathbf{C}\right) \text { DSC } \\
\text { peak }\end{array}$ & $\mathrm{T}\left({ }^{\circ} \mathrm{C}\right) 10 \%$ mass loss \\
\hline Glycerol & 269 & 204 \\
\hline $25 \%[\mathrm{C} 2 \mathrm{mim}][\mathrm{O} 2 \mathrm{CH}]$ & 267 & 221 \\
\hline $50 \%[\mathrm{C} 2 \mathrm{mim}][\mathrm{O} 2 \mathrm{CH}]$ & 270 & 233 \\
\hline $75 \%[\mathrm{C} 2 \mathrm{mim}][\mathrm{O} 2 \mathrm{CH}]$ & 274 & 231 \\
\hline $100 \%[\mathrm{C} 2 \mathrm{mim}][\mathrm{O} 2 \mathrm{CH}]$ & 258 & 206 \\
\hline $25 \%[\mathrm{C} 2 \mathrm{mim}][\mathrm{OAc}]$ & 290 & 224 \\
\hline $50 \%[$ C2mim $][\mathrm{OAc}]$ & 290 & 245 \\
\hline 75\% [C2mim] $[\mathrm{OAc}]$ & 281 & 244 \\
\hline $100 \%[\mathrm{C} 2 \mathrm{mim}][\mathrm{OAc}]$ & 257 & 228 \\
\hline
\end{tabular}

\section{References}

1. $\quad$ Goering, H. K.; Soest, P. J., Forage fiber analysis. USDA Agric. Handb. 1970, 379, 1-9. 2. $\quad$ Raj, T.; Kapoor, M.; Gaur, R.; Christopher, J.; Lamba, B.; Tuli, D. K.; Kumar, R., Physical and Chemical Characterization of Various Indian Agriculture Residues for Biofuels Production. Energy Fuels 2015, 29 (5), 3111-3118.

3. Wang, F.; Niu, W.; Zhang, A.; Yi, W., Enhanced anaerobic digestion of corn stover by thermo-chemical pretreatment. Int. J. Agric.\& Biol. Eng. 2015, 8 (1), 84-90.

4. Li, J.; Zhang, R.; Siddhu, M. A. H.; He, Y.; Wang, W.; Li, Y.; Chen, C.; Liu, G., Enhancing methane production of corn stover through a novel way: Sequent pretreatment of potassium hydroxide and steam explosion. Bioresour. Tech. 2015, 181, 345-350.

5. $\quad$ Lin, X.; Sui, S.; Tan, S.; Pittman, C. U., Jr.; Sun, J.; Zhang, Z., Fast Pyrolysis of Four Lignins from Different Isolation Processes Using Py-GC/MS. Energies 2015, 8 (6), 5107-5121.

6. Costa, L. A. D. S.; Fonseca, A. F.; Pereira, F. V.; Druzian, J. I., EXTRACTION AND CHARACTERIZATION OF CELLULOSE NANOCRYSTALS FROM CORN STOVER. Cell Chem. Technol. 2015, 49 (2), 127-133. 
7. Lopes, A. M. D.; João, K. G.; Rubik, D. F.; Bogel-Łukasik, E.; Duarte, L. C.; Andreaus, J.; Bogel-Łukasik, R., Pre-treatment of lignocellulosic biomass using ionic liquids: Wheat straw fractionation. Bioresour. Technol. 2013, 142, 198-208.

8. Wang, Q.; Wei, W.; Li, X.; Sun, J.; He, J.; He, M., Comparative Study of Alkali and Acidic Cellulose Solvent Pretreatment of Corn Stover for Fermentable Sugar Production. Bioresources 2016, 11 (1), 482-491.

9. Colom, X.; Carrillo, F.; Nogues, F.; Garriga, P., Structural analysis of photodegraded wood by means of FTIR spectroscopy. Polym. Degrad. Stabil. 2003, 80 (3), 543-549.

10. Zhao, B.; Greiner, L.; Leitner, W., Cellulose solubilities in carboxylate-based ionic liquids. RSC Adv. 2012, 2 (6), 2476-2479.

11. Lynam, J. G.; Coronella, C. J., Glycerol as an ionic liquid co-solvent for pretreatment of rice hulls to enhance glucose and xylose yield. Bioresour.Technol. 2014, 166, 471-478.

12. Lynam, J. G. Biomass Pretreatment using Ionic Liquid and Glycerol Mixtures.

Dissertation, University of Nevada, Reno, Reno, Nevada, 2015.

13. Dagdug, L., A theoretical framework for the Vogel-Fulcher-Tammann equation for covalent network glasses derived by the stochastic matrix method. J. Phys. Condens. Matter 2000, 12 (46), 9573-9589.

14. Vranes, M.; Tot, A.; Papovic, S.; Zec, N.; Dozic, S.; Gadzuric, S., Ideal and non-ideal behaviour of \{1-butyl-1-methylpyrrolydinium bis(trifluoromethylsulfonyl)imide plus gammabutyrolactone \} binary mixtures. J. Chem. Thermodyn. 2015, 81, 66-76. 